\title{
ON SOME PROPERTIES OF A GENERAL CLASS OF TWO-PIECE SKEW NORMAL DISTRIBUTION
}

\author{
C. Satheesh Kumar* and M. R. Anusree*
}

\begin{abstract}
A new class of generalized two-piece skew normal distribution is introduced here as a two-piece version of the generalized skew normal distribution of Kumar and Anusree (2011). It is shown that the proposed class of distribution will be more suitable for modelling skewed, multimodal data sets. Several properties of the model are studied and the maximum likelihood estimation of the parameters of the distribution is discussed. Further, the practical usefulness of the model is illustrated with the help of certain real life data sets.
\end{abstract}

Key words and phrases: Asymmetric distributions, method of maximum likelihood, plurimodality, reliability measures, skew normal distribution.

\section{Introduction}

An important drawback of the normal distribution from a practical point of view is its symmetric behaviour. Azzalini (1985) provided a methodology to introduce skewness in a normal distribution and he defined the skew normal distribution as follows:

A random variable $\mathrm{X}$ is said to have the skew normal distribution with skewness parameter $\lambda \in R=(-\infty, \infty)$, denoted by $S N D(\lambda)$, if its probability density function (p.d.f.) is of the following form, for $x \in R$.

$$
g_{1}(x ; \lambda)=2 f(x) F(\lambda x)
$$

where $f(\cdot)$ and $F(\cdot)$ are respectively the p.d.f. and cumulative distribution function (c.d.f.) of a standard normal variate. The $S N D(\lambda)$ has been studied by Azzalini (1985, 1986), Henze (1986) and several others. Further, various generalizations were studied by Azzalini and Dalla Valle (1996), Mudholkar and Hutson (2000), Branco and Dey (2001), Arnold and Beaver (2002), Arellano-Valle et al. (2004), Sharafi and Behboodian (2008), Jamalizadeh et al. (2008, 2009), and Kumar and Anusree (2011, 2013a, b, 2014a, b).

Kim (2005) introduced a two-piece version of the $S N D(\lambda)$ through the following p.d.f.

$$
g_{K}(x ; \lambda)=\frac{2 \pi f(x) F(\lambda|x|)}{\left(\pi+2 \tan ^{-1}(\lambda)\right)} .
$$

The distribution of a random variable with p.d.f. (1.2) hereafter we denote as $\operatorname{TSND}_{K}(\lambda)$. It is seen that there exists a symmetric behaviour in $\operatorname{TSND}_{K}(\lambda)$

\footnotetext{
Received August 12, 2014. Revised December 5, 2014. Accepted December 31, 2014.

*Department of Statistics, University of Kerala, Trivandrum 695 581, India. Email: drcsatheeshkumar@gmail.com, anusreemr@yahoo.co.in
} 
as determined by the sign of $\lambda$ on either side of the origin. In order to mitigate this limitation, Kumar and Anusree (2013a) introduced a wide class of the twopiece skew normal distribution denoted by $\operatorname{TSND}_{K A}(\lambda, \rho)$, through the following p.d.f., in which $\rho \in[-1,1]$ and $C=2 \pi\left[\pi-\tan ^{-1}(\lambda)+\tan ^{-1}(\rho \lambda)\right]^{-1}$.

$$
g_{K A}(x ; \lambda, \rho)= \begin{cases}C f(x) F(\lambda x), & x<0 \\ C f(x) F(\rho \lambda x), & x \geq 0 .\end{cases}
$$

Note that $\operatorname{TSND}_{K A}(-\lambda,-1)$ is $T S N D_{K}(\lambda)$. In order to accomodate plurimodality, Kumar and Anusree (2011) developed a generalized version of the $S N D(\lambda)$ by considering a mixture of the standard normal and $S N D(\lambda)$ through the following p.d.f., in which $x \in R, \lambda \in R$ and $\alpha \geq-1$.

$$
g_{2}(x ; \lambda, \alpha)=\frac{2}{(\alpha+2)} f(x)[1+\alpha F(\lambda x)] .
$$

A distribution with p.d.f. (1.4) they notated as the $\operatorname{GMNSND}(\lambda, \alpha)$. In order to develop a more flexible class of asymmetric, plurimodal normal type distributions, through this paper we propose a two-piece version of the $\operatorname{GMNSND}(\lambda, \alpha)$ and named it "the extended two-piece skew normal distribution" or in short "the ETPSND". The ETPSND reduces to the standard normal distribution, the $\operatorname{SND}(\lambda)$ of Azzalini (1985), the $\operatorname{GMNSND}(\lambda, \alpha)$, the two-piece skew normal distribution of Kim (2005) and the generalized two-piece skew normal distribution of Kumar and Anusree (2013a) for particular choices of its shape parameters as its special cases. The rest of the paper is organized as follows: The definition of ETPSND and some of its important properties are given in Section 2. In Section 3, we obtain expressions for certain reliability measures and discuss also some concepts regarding the mode of the distribution. In Section 4, a locationscale extension of the ETPSND is suggested and some of its important structural properties are studied. Finally, the maximum likelihood estimation of the parameters is discussed in Section 5 and its practical usefulness is illustrated with the help of certain real life data sets.

We need the following notation in the sequel. For any $a \in R$ and $b>0$, define

$$
\xi(a, b)=\int_{a}^{\infty} f(x) \int_{0}^{b x} f(y) d y d x
$$

so that

$$
\xi(0, \lambda)=\frac{\tan ^{-1}(\lambda)}{2 \pi}
$$

and for any reals $a, b$ and $s$ such that $b x+s>0$,

$$
\xi_{s}(a, b)=\int_{a}^{\infty} f(x) \int_{0}^{b x+s} f(y) d y d x .
$$




\section{Definition and properties}

First we present the definition of the extended two-piece skew normal distribution and discuss some of its properties.

Definition 2.1. A random variable $Z$ is said to follow the extended twopiece skew normal distribution with parameters $\lambda_{1}, \lambda_{2} \in R$ and $\alpha \geq-1$ if its p.d.f. $h\left(z ; \lambda_{1}, \lambda_{2}, \alpha\right)$ is of the following form. For $z \in R$,

$$
h\left(z ; \lambda_{1}, \lambda_{2}, \alpha\right)= \begin{cases}\frac{2}{(\alpha+2)} f(z)\left[1+D\left(\lambda_{1}, \lambda_{2}, \alpha\right) F\left(\lambda_{1} z\right)\right], & z<0 \\ \frac{2}{(\alpha+2)} f(z)\left[1+D\left(\lambda_{1}, \lambda_{2}, \alpha\right) F\left(\lambda_{2} z\right)\right], & z \geq 0\end{cases}
$$

where $D\left(\lambda_{1}, \lambda_{2}, \alpha\right)=\alpha \pi\left[\pi+\tan ^{-1}\left(\lambda_{2}\right)-\tan ^{-1}\left(\lambda_{1}\right)\right]^{-1}$.

Here $D\left(\lambda_{1}, \lambda_{2}, \alpha\right)$ is obtained by using Lemmas 2.1 and 2.2 as given in Kumar and Anusree (2013a). The distribution of a random variable $Z$ with p.d.f. (2.1) we denoted as $\operatorname{ETPSND}\left(\lambda_{1}, \lambda_{2}, \alpha\right)$.

For some particular choices of $\lambda_{1}, \lambda_{2}$ and $\alpha$ the p.d.f. given in (2.1) of $\operatorname{ETPSND}\left(\lambda_{1}, \lambda_{2}, \alpha\right)$ is plotted and is shown in Fig. 1.

Some important special cases of the $\operatorname{ETPSND}\left(\lambda_{1}, \lambda_{2}, \alpha\right)$ are

(i) $\operatorname{ETPSND}\left(\lambda_{1}, \lambda_{2}, 0\right), \operatorname{ETPSND}(0,0, \alpha)$ or the limiting case of the $\operatorname{ETPSND}\left(\lambda_{1}, \lambda_{2}, \alpha\right)$ when $\lambda_{1} \rightarrow-\infty, \lambda_{2} \rightarrow \infty$, is the standard normal distribution,

(ii) the limiting case of the $\operatorname{ETPSND}\left(\lambda_{1}, \lambda_{2}, \alpha\right)$ when $\alpha \rightarrow \infty$ is the $\operatorname{TSND}_{K A}(\lambda, \rho)$,

(iii) the limiting case of the $\operatorname{ETPSND}(\lambda, \lambda, \alpha)$ when $\alpha \rightarrow \infty$ is the $\operatorname{TSND}_{K}(\lambda)$,

(iv) the $\operatorname{ETPSND}(\lambda, \lambda, \alpha)$ is the $\operatorname{GMNSND}(\lambda, \alpha)$, which reduces to $\operatorname{SND}(\lambda)$ when $\alpha=-1$ and

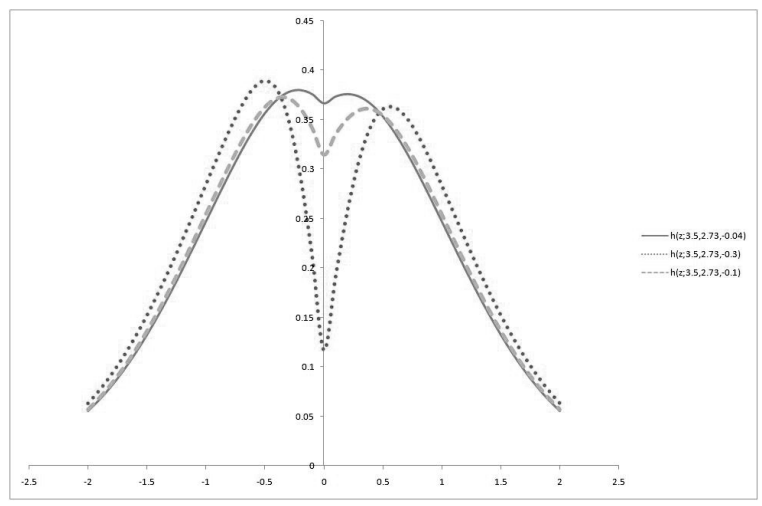

Figure 1. Probability plots of $\operatorname{ETPSND}\left(\lambda_{1}, \lambda_{2}, \alpha\right)$ for different choices of $\alpha$. 
(v) the limiting case of the $\operatorname{ETPSND}\left(\lambda_{1}, \lambda_{2}, \alpha\right)$ as $\lambda_{1} \rightarrow \infty, \lambda_{2} \rightarrow \infty$ and $\alpha \rightarrow \infty$ or $\lambda_{1} \rightarrow-\infty, \lambda_{2} \rightarrow-\infty$ and $\alpha \rightarrow \infty$ is the standard half normal distribution.

We obtain certain structural properties of the $\operatorname{ETPSND}\left(\lambda_{1}, \lambda_{2}, \alpha\right)$ through the following results.

RESUlt 2.1. If $Z$ follows $\operatorname{ETPSND}\left(\lambda_{1}, \lambda_{2}, \alpha\right)$ with p.d.f. $h\left(z ; \lambda_{1}, \lambda_{2}, \alpha\right)$, then $Y_{1}=-Z$ follows $\operatorname{ETPSND}\left(-\lambda_{2},-\lambda_{1}, \alpha\right)$.

Proof. For any $y_{1} \in R$, the p.d.f. $h_{1}\left(y_{1} ; \lambda_{1}, \lambda_{2}, \alpha\right)$ of $Y_{1}$ is given by

$$
\begin{aligned}
h_{1}\left(y_{1} ; \lambda_{1}, \lambda_{2}, \alpha\right) & =h\left(-y_{1} ; \lambda_{1}, \lambda_{2}, \alpha\right)\left|\frac{d z}{d y_{1}}\right| \\
& = \begin{cases}\frac{2}{(\alpha+2)} f\left(y_{1}\right)\left[1+D\left(\lambda_{1}, \lambda_{2}, \alpha\right) F\left(-\lambda_{2} y_{1}\right)\right], & y_{1}<0 \\
\frac{2}{(\alpha+2)} f\left(y_{1}\right)\left[1+D\left(\lambda_{1}, \lambda_{2}, \alpha\right) F\left(-\lambda_{1} y_{1}\right)\right], & y_{1} \geq 0,\end{cases}
\end{aligned}
$$

which shows that $Y_{1}=-Z$ follows $\operatorname{ETPSND}\left(-\lambda_{2},-\lambda_{1}, \alpha\right)$.

RESUlt 2.2. If $Z$ follows $\operatorname{ETPSND}\left(\lambda_{1}, \lambda_{2}, \alpha\right)$ with p.d.f. $h\left(z ; \lambda_{1}, \lambda_{2}, \alpha\right)$, then $Y_{2}=Z^{2}$ has p.d.f. (2.2).

Proof. The p.d.f. $h_{2}\left(y_{2} ; \lambda_{1}, \lambda_{2}, \alpha\right)$ of $Y_{2}$ is given by

$$
\begin{aligned}
h_{2}\left(y_{2} ; \lambda_{1}, \lambda_{2}, \alpha\right)= & h\left(z ; \lambda_{1}, \lambda_{2}, \alpha\right)\left|\frac{d z}{d y_{2}}\right| \\
= & h\left(-\sqrt{y_{2}} ; \lambda_{1}, \lambda_{2}, \alpha\right)\left|\frac{d z}{d y_{2}}\right|+h\left(\sqrt{y_{2}} ; \lambda_{1}, \lambda_{2}, \alpha\right)\left|\frac{d z}{d y_{2}}\right| \\
= & \frac{1}{(\alpha+2)}\left(\frac{f\left(\sqrt{y_{2}}\right)}{2 \sqrt{y_{2}}}\right) \\
& \times\left[2+D\left(\lambda_{1}, \lambda_{2}, \alpha\right)\left(F\left(-\lambda_{1} \sqrt{y_{2}}\right)+F\left(\lambda_{2} \sqrt{y_{2}}\right)\right)\right] .
\end{aligned}
$$

Remark 2.1. When $\lambda_{1}=\lambda_{2}$ and $\alpha=-1$, the p.d.f. given in (2.2) reduces to the p.d.f. of a Chi-square variate with one degree of freedom.

In order to find the distribution function of $\operatorname{ETPSND}\left(\lambda_{1}, \lambda_{2}, \alpha\right)$, we need the following result.

RESUlt 2.3. If $Z$ is a $\operatorname{ETPSND}\left(\lambda_{1}, \lambda_{2}, \alpha\right)$, then for any real $d_{1}, d_{2}$ such that $d_{1} \leq d_{2}$, where $\xi(a, b)$ is as given in (1.5).

(2.3) $\quad P\left(d_{1} \leq Z \leq d_{2}\right)$ 


$$
=\left\{\begin{array}{l}
\frac{2}{(\alpha+2)}\left[F\left(d_{2}\right)-F\left(d_{1}\right)\right]+\frac{D\left(\lambda_{1}, \lambda_{2}, \alpha\right)}{(\alpha+2)}\left[F\left(d_{2}\right)-F\left(d_{1}\right)\right] \\
\quad+\frac{D\left(\lambda_{1}, \lambda_{2}, \alpha\right)}{(\alpha+2)}\left[2 \xi\left(d_{1}, \lambda_{1}\right)-2 \xi\left(d_{2}, \lambda_{1}\right)\right], \quad d_{1} \leq d_{2}<0 \\
\frac{2}{(\alpha+2)}\left[F\left(d_{2}\right)-F\left(d_{1}\right)\right]+\frac{D\left(\lambda_{1}, \lambda_{2}, \alpha\right)}{(\alpha+2)}\left[F\left(d_{2}\right)-F\left(d_{1}\right)\right] \\
+\frac{D\left(\lambda_{1}, \lambda_{2}, \alpha\right)}{(\alpha+2)}\left[2 \xi\left(d_{1}, \lambda_{2}\right)-2 \xi\left(d_{2}, \lambda_{2}\right)\right], \quad 0 \leq d_{1} \leq d_{2} .
\end{array}\right.
$$

Proof. For any $d_{1} \leq d_{2}<0$, by definition,

$$
\begin{aligned}
P\left(d_{1} \leq Z \leq d_{2}\right)= & \int_{d_{1}}^{d_{2}} h\left(z ; \lambda_{1}, \lambda_{2}, \alpha\right) d z \\
= & \int_{d_{1}}^{d_{2}}\left[\frac{2}{(\alpha+2)} f(z)+\frac{D\left(\lambda_{1}, \lambda_{2}, \alpha\right)}{(\alpha+2)} 2 f(z) F\left(\lambda_{1} z\right)\right] d z \\
= & \frac{2}{(\alpha+2)}\left[F\left(d_{2}\right)-F\left(d_{1}\right)\right] \\
& +\frac{D\left(\lambda_{1}, \lambda_{2}, \alpha\right)}{(\alpha+2)}\left[G\left(d_{2}, \lambda_{1}\right)-G\left(d_{1}, \lambda_{1}\right)\right]
\end{aligned}
$$

where $G(\cdot, \lambda)$ is the distribution function of the $S N D(\lambda)$.

Now, for the case $0 \leq d_{1} \leq d_{2}$,

$$
\begin{aligned}
P\left(d_{1} \leq Z \leq d_{2}\right)= & \int_{d_{1}}^{d_{2}} h\left(z ; \lambda_{1}, \lambda_{2}, \alpha\right) d z \\
= & \int_{d_{1}}^{d_{2}}\left[\frac{2}{(\alpha+2)} f(z)+\frac{D\left(\lambda_{1}, \lambda_{2}, \alpha\right)}{(\alpha+2)} 2 f(z) F\left(\lambda_{2} z\right)\right] d z \\
= & \frac{2}{(\alpha+2)}\left[F\left(d_{2}\right)-F\left(d_{1}\right)\right] \\
& +\frac{D\left(\lambda_{1}, \lambda_{2}, \alpha\right)}{(\alpha+2)}\left[G\left(d_{2}, \lambda_{2}\right)-G\left(d_{1}, \lambda_{2}\right)\right] .
\end{aligned}
$$

Thus (2.4) and (2.5) implies (2.3).

RESUlt 2.4. The c.d.f. $H(z)$ of a random variable $Z$ following $\operatorname{ETPSND}\left(\lambda_{1}, \lambda_{2}, \alpha\right)$ with p.d.f. (2.1) is the following, in which $\xi(a, b)$ is as given in (1.5).

$$
H(z)=\frac{2}{(\alpha+2)}\left\{\begin{array}{cc}
F(z)+\frac{D\left(\lambda_{1}, \lambda_{2}, \alpha\right)}{2}\left[F(z)-2 \xi\left(z, \lambda_{1}\right)\right], & z<0 \\
F(z)+\frac{D\left(\lambda_{1}, \lambda_{2}, \alpha\right)}{2}\left[F(z)-2 \xi\left(z, \lambda_{2}\right)\right. & \\
\left.-\frac{\tan ^{-1}\left(\lambda_{1}\right)}{\pi}+\frac{\tan ^{-1}\left(\lambda_{2}\right)}{\pi}\right], & z \geq 0 .
\end{array}\right.
$$


Proof. $\quad$ Let $Z$ be a random variable with p.d.f. (2.1) and c.d.f. $H(z)$. Then by definition,

$$
H(z)= \begin{cases}L_{1}, & z<0 \\ L_{2}, & z \geq 0\end{cases}
$$

where

$$
\begin{aligned}
L_{1} & =\frac{2}{(\alpha+2)} \int_{-\infty}^{z} f(t) d t+\frac{D\left(\lambda_{1}, \lambda_{2}, \alpha\right)}{(\alpha+2)} \int_{-\infty}^{z} 2 f(t) F\left(\lambda_{1} t\right) d t \\
& =\frac{2}{(\alpha+2)} F(z)+\frac{D\left(\lambda_{1}, \lambda_{2}, \alpha\right)}{(\alpha+2)} G\left(z, \lambda_{1}\right) \\
& =\frac{2}{(\alpha+2)} F(z)+\frac{D\left(\lambda_{1}, \lambda_{2}, \alpha\right)}{(\alpha+2)}\left[F(z)-2 \xi\left(z, \lambda_{1}\right)\right]
\end{aligned}
$$

and

$$
\begin{aligned}
L_{2}= & \int_{-\infty}^{0} \frac{2}{(\alpha+2)} f(t)\left[1+D\left(\lambda_{1}, \lambda_{2}, \alpha\right) F\left(\lambda_{1} t\right)\right] d t \\
& +\int_{0}^{z} \frac{2}{(\alpha+2)} f(t)\left[1+D\left(\lambda_{1}, \lambda_{2}, \alpha\right) F\left(\lambda_{2} t\right)\right] d t \\
= & \frac{2}{(\alpha+2)}\left[F(z)+\frac{D\left(\lambda_{1}, \lambda_{2}, \alpha\right)}{2} G\left(0, \lambda_{1}\right)\right. \\
& \left.\quad+\frac{D(\lambda, \alpha, \rho)}{2}\left(G\left(z, \lambda_{2}\right)-G\left(0, \lambda_{2}\right)\right)\right] \\
= & \frac{2}{(\alpha+2)}\left[F(z)+\frac{D\left(\lambda_{1}, \lambda_{2}, \alpha\right)}{2}\left(\frac{1}{2}-2 \xi\left(0, \lambda_{1}\right)\right)\right. \\
& \left.\quad+\frac{D\left(\lambda_{1}, \lambda_{2}, \alpha\right)}{2}\left(F(z)-2 \xi\left(z, \lambda_{2}\right)-\frac{1}{2}-2 \xi\left(0, \lambda_{2}\right)\right)\right] .
\end{aligned}
$$

Since $\xi(0, \cdot)$ is as defined in (1.6), on substituting (2.8) and (2.9) in (2.7) we get $(2.6)$.

RESUlt 2.5. The characteristic function, $\phi_{Z}(t)$ of a random variable $Z$ following $\operatorname{ETPSND}\left(\lambda_{1}, \lambda_{2}, \alpha\right)$ with p.d.f. (2.1) is the following, for any $t \in R$ and $i^{2}=-1$.

$$
\begin{aligned}
\phi_{Z}(t)= & \frac{2}{(\alpha+2)} e^{-t^{2} / 2}\left[1+D\left(\lambda_{1}, \lambda_{2}, \alpha\right) F\left(i \delta_{1} t\right)\right] \\
& -\frac{2}{(\alpha+2)} D\left(\lambda_{1}, \lambda_{2}, \alpha\right) e^{-t^{2} / 2}\left[\xi_{k_{1}}\left(-i t, \lambda_{1}\right)-\xi_{k_{2}}\left(-i t, \lambda_{2}\right)\right]
\end{aligned}
$$

where, $\delta_{1}=\frac{\lambda_{1}}{\sqrt{1+\lambda_{1}^{2}}}$ for $j=1,2$ and $\xi_{s}(a, b)$ is defined in (1.7). 
Proof. Let $Z$ follow $\operatorname{ETPSND}\left(\lambda_{1}, \lambda_{2}, \alpha\right)$ with p.d.f. (2.1). By the definition of the characteristic function, for any $t \in R$ we have

$$
\begin{aligned}
\phi_{Z}(t) & =E\left(e^{i t Z}\right) \\
& =\int_{-\infty}^{\infty} e^{i t z} h\left(z ; \lambda_{1}, \lambda_{2}, \alpha\right) d z .
\end{aligned}
$$

On substituting (2.1) in (2.11) and rearranging the terms in light of the expression for the characteristic function of a standard normal variate, we obtain the following,

$$
\begin{aligned}
\phi_{Z}(t)=\frac{2}{(\alpha+2)} e^{-t^{2} / 2}\{ & 1+D\left(\lambda_{1}, \lambda_{2}, \alpha\right) \int_{-\infty}^{\infty} \frac{1}{\sqrt{2 \pi}} e^{-(z-i t)^{2} / 2} F\left(\lambda_{1} z\right) d z \\
& -D\left(\lambda_{1}, \lambda_{2}, \alpha\right) \int_{0}^{\infty} \frac{1}{\sqrt{2 \pi}} e^{-(z-i t)^{2} / 2} F\left(\lambda_{1} z\right) d z \\
& \left.+D\left(\lambda_{1}, \lambda_{2}, \alpha\right) \int_{0}^{\infty} \frac{1}{\sqrt{2 \pi}} e^{-(z-i t)^{2} / 2} F\left(\lambda_{2} z\right) d z\right\}
\end{aligned}
$$

If we put $z-i t=x$ in (2.12), we get

$$
\begin{aligned}
\phi_{Z}(t)= & \frac{2}{(\alpha+2)} e^{-t^{2} / 2}\left[1+D\left(\lambda_{1}, \lambda_{2}, \alpha\right) \int_{-\infty}^{\infty} \frac{e^{-x^{2} / 2} F\left(\lambda_{1}(x+i t)\right) d x}{\sqrt{2 \pi}}\right] \\
& -D\left(\lambda_{1}, \lambda_{2}, \alpha\right) \int_{-i t}^{\infty} \frac{e^{-x^{2} / 2} F\left(\lambda_{1}(x+i t)\right) d x}{\sqrt{2 \pi}} \\
& +D\left(\lambda_{1}, \lambda_{2}, \alpha\right) \int_{-i t}^{\infty} \frac{e^{-x^{2} / 2} F\left(\lambda_{2}(x+i t)\right) d x}{\sqrt{2 \pi}} \\
= & \frac{2}{(\alpha+2)} e^{-t^{2} / 2}\left\{1+D\left(\lambda_{1}, \lambda_{2}, \alpha\right) F\left(i \delta_{1} t\right)\right. \\
& -D\left(\lambda_{1}, \lambda_{2}, \alpha\right) \int_{-i t}^{\infty} f(x) F\left(\lambda_{1}(x+i t)\right) d x \\
& \left.+D\left(\lambda_{1}, \lambda_{2}, \alpha\right) \int_{-i t}^{\infty} f(x) F\left(\lambda_{2}(x+i t)\right) d x\right\},
\end{aligned}
$$

in light of lemma 2.1 of Kumar and Anusree (2013a). Now, on rearranging the terms and putting $k=\lambda i t$ to arrive at the following.

$$
\begin{aligned}
\phi_{Z}(t)= & \frac{2}{(\alpha+2)} e^{-t^{2} / 2}\left[1+D\left(\lambda_{1}, \lambda_{2}, \alpha\right) F\left(i \delta_{1} t\right)\right] \\
& -\frac{D\left(2 \lambda_{1}, \lambda_{2}, \alpha\right)}{(\alpha+2)} e^{-t^{2} / 2} \int_{-i t}^{\infty} f(x)\left[\int_{-\infty}^{0} f(u) d u+\int_{0}^{\lambda_{1} x+k_{1}} f(u) d u\right] d x \\
& +\frac{2 D\left(\lambda_{1}, \lambda_{2}, \alpha\right)}{(\alpha+2)} e^{-t^{2} / 2} \int_{-i t}^{\infty} f(x)\left[\int_{-\infty}^{0} f(u) d u+\int_{0}^{\lambda_{2} x+\rho k_{2}} f(u) d u\right] d x,
\end{aligned}
$$

which implies (2.10), in light of (1.7). 


\section{Reliability measures and mode}

Here we present some reliability aspects of the $\operatorname{ETPSND}\left(\lambda_{1}, \lambda_{2}, \alpha\right)$ with p.d.f. (2.1) and discuss some concepts regarding its mode.

RESUlT 3.1. The reliability function $R(t)$ of a random variable $Z$ having the $\operatorname{ETPSND}\left(\lambda_{1}, \lambda_{2}, \alpha\right)$ is the following, in which $\xi(t, \cdot)$ is as defined in (1.5).

$$
R(t)=1-\frac{2}{(\alpha+2)}\left\{\begin{array}{rlrl}
F(t) & +\frac{D\left(\lambda_{1}, \lambda_{2}, \alpha\right)}{2}\left[F(t)-2 \xi\left(t, \lambda_{1}\right)\right], & & t<0 \\
F(t) & +\frac{D\left(\lambda_{1}, \lambda_{2}, \alpha\right)}{2}\left[F(t)-2 \xi\left(t, \lambda_{2}\right)\right] \\
& +\frac{D\left(\lambda_{1}, \lambda_{2}, \alpha\right)}{2} \\
& \times\left[\frac{-1}{\pi} \tan ^{-1}\left(\lambda_{1}\right)+\frac{1}{\pi} \tan ^{-1}\left(\lambda_{2}\right)\right], \quad t \geq 0 .
\end{array}\right.
$$

Proof follows from the definition of the reliability function $R(t)=1-H(t)$, where $H(\cdot)$ is the c.d.f. of $Z$ as obtained in Result 2.4 .

RESUlt 3.2. The failure rate $r(t)$ of a random variable $Z$ following the $\operatorname{ETPSND}\left(\lambda_{1}, \lambda_{2}, \alpha\right)$ with p.d.f. $h\left(z ; \lambda_{1}, \lambda_{2}, \alpha\right)$ is

$$
r(t)=\left\{\begin{array}{cc}
\frac{2 f(t)\left[1+D\left(\lambda_{1}, \lambda_{2}, \alpha\right) F\left(\lambda_{1} t\right)\right]}{(\alpha+2)-2\left(F(t)+D\left(\lambda_{1}, \lambda_{2}, \alpha\right)\left[F(t)-2 \xi\left(t, \lambda_{1}\right)\right]\right)}, & t<0 \\
\frac{2 f(t)\left[1+D\left(\lambda_{1}, \lambda_{2}, \alpha\right) F\left(\lambda_{2} t\right)\right]}{(\alpha+2)-2\left(F(t)+D\left(\lambda_{1}, \lambda_{2}, \alpha\right)\left[F(t)-2 \xi\left(t, \lambda_{2}\right)-\frac{1}{\pi} \tan ^{-1}\left(\lambda_{1}\right)+\frac{1}{\pi} \tan ^{-1}\left(\lambda_{2}\right)\right]\right)} \\
t \geq 0 .
\end{array}\right.
$$

Proof follows from the definition of failure rate, $r(t)=\frac{h\left(t ; \lambda_{1}, \lambda_{2}, \alpha\right)}{R(t)}$, where $R(t)$ is the reliability function as given in (3.1).

REsult 3.3. The mean residual life function(MRLF) $\mu(t)$ of $\operatorname{ETPSND}\left(\lambda_{1}, \lambda_{2}, \alpha\right)$ is the following, in which $\Delta=\frac{2}{(\alpha+2) R(t)}, \delta_{j}=\frac{\lambda_{j}}{\sqrt{1+\lambda_{j}^{2}}}$ for $j=1.2$.

$$
\mu(t)=\Delta\left\{\begin{array}{rlrl}
f(t) & +D\left(\lambda_{1}, \lambda_{2}, \alpha\right) f(t) F\left(\lambda_{1} t\right)+\frac{\delta_{1}}{2 \sqrt{2 \pi}} D\left(\lambda_{1}, \lambda_{2}, \alpha\right) & & \\
& -\frac{\delta_{1}}{\sqrt{2 \pi}} D\left(\lambda_{1}, \lambda_{2}, \alpha\right) F\left(t \sqrt{1+\lambda_{1}^{2}}\right) & \\
& +\frac{\delta_{2}}{2 \sqrt{2 \pi}} D\left(\lambda_{1}, \lambda_{2}, \alpha\right)-t, & & t \leq 0 \\
f(t) & +D\left(\lambda_{1}, \lambda_{2}, \alpha\right) f(t) F\left(\lambda_{2} t\right)+\frac{\delta_{2}}{2 \sqrt{2 \pi}} D\left(\lambda_{1}, \lambda_{2}, \alpha\right) & \\
& -\frac{\delta_{2}}{\sqrt{2 \pi}} D\left(\lambda_{1}, \lambda_{2}, \alpha\right) F\left(t \sqrt{1+\lambda_{2}^{2}}\right)-t, & t>0
\end{array}\right.
$$


Proof. By definition, the mean residual life function of $Z$ following the $\operatorname{ETPSND}\left(\lambda_{1}, \lambda_{2}, \alpha\right)$ is given by

$$
\begin{aligned}
\mu(t)= & E(Z \mid Z>t)-t \\
= & \frac{2}{R(t)(\alpha+2)} \\
& \times\left\{\begin{array}{rr}
\int_{t}^{0} z f(z)\left[1+D\left(\lambda_{1}, \lambda_{2}, \alpha\right) F\left(\lambda_{1} z\right)\right] d z \\
\quad+\int_{0}^{\infty} z f(z) d z \\
\quad+D\left(\lambda_{1}, \lambda_{2}, \alpha\right) \int_{0}^{\infty} z f(z) F\left(\lambda_{2} z\right) d z-t, & t \leq 0 \\
\int_{t}^{\infty} z f(z)\left[1+D\left(\lambda_{1}, \lambda_{2}, \alpha\right) F\left(\lambda_{2} z\right)\right] d z-t, & t>0 .
\end{array}\right.
\end{aligned}
$$

Now for any $t<0$,

$$
\begin{array}{rl}
\int_{t}^{0} z & f(z)\left[1+D\left(\lambda_{1}, \lambda_{2}, \alpha\right) F\left(\lambda_{1} z\right)\right] d z \\
= & -\int_{t}^{0} f^{\prime}(z)\left[1+D\left(\lambda_{1}, \lambda_{2}, \alpha\right) F\left(\lambda_{1} z\right)\right] d z \\
= & f(t)-\frac{1}{\sqrt{2 \pi}}-D\left(\lambda_{1}, \lambda_{2}, \alpha\right)\left[\frac{1}{2 \sqrt{(2 \pi)}}-F\left(\lambda_{1} t\right) f(t)\right] \\
& -D\left(\lambda_{1}, \lambda_{2}, \alpha\right) \lambda_{1} \int_{t}^{0} f\left(\lambda_{1} z\right) f(z) d z \\
= & f(t)-\frac{1}{\sqrt{2 \pi}}-\frac{D\left(\lambda_{1}, \lambda_{2}, \alpha\right)}{2 \sqrt{2 \pi}}+D\left(\lambda_{1}, \lambda_{2}, \alpha\right) F\left(\lambda_{1} t\right) f(t) \\
& +\frac{\lambda_{1} D\left(\lambda_{1}, \lambda_{2}, \alpha\right)\left[\frac{1}{2}-F\left(t \sqrt{1+\lambda_{1}^{2}}\right)\right]}{\sqrt{2 \pi} \sqrt{1+\lambda_{1}^{2}}}
\end{array}
$$

and for any $t>0$,

$$
\begin{aligned}
\int_{t}^{\infty} & z f(z)\left[1+D\left(\lambda_{1}, \lambda_{2}, \alpha\right) F\left(\lambda_{2} z\right)\right] d z \\
= & -\int_{t}^{\infty} f^{\prime}(z)\left[1+D\left(\lambda_{1}, \lambda_{2}, \alpha\right) F\left(\lambda_{2} z\right)\right] d z \\
= & f(t)+D\left(\lambda_{1}, \lambda_{2}, \alpha\right) F\left(\lambda_{2} t\right) f(t)+\frac{\lambda_{2} D\left(\lambda_{1}, \lambda_{2}, \alpha\right)}{\sqrt{2 \pi} \sqrt{1+\lambda_{2}^{2}}} \\
& -D\left(\lambda_{1}, \lambda_{2}, \alpha\right) \frac{\lambda_{2}}{\sqrt{2 \pi} \sqrt{1+\lambda_{2}^{2}}} F\left(t \sqrt{1+\lambda_{2}^{2}}\right) .
\end{aligned}
$$

In particular, when $t=0$ in (3.6) we have

$$
\begin{aligned}
\int_{0}^{\infty} z f(z)\left[1+D\left(\lambda_{1}, \lambda_{2}, \alpha\right) F\left(\lambda_{2} z\right)\right] d z= & \frac{1}{\sqrt{(2 \pi)}}+\frac{D\left(\lambda_{1}, \lambda_{2}, \alpha\right)}{2 \sqrt{2 \pi}} \\
& +\frac{\lambda_{2} D\left(\lambda_{1}, \lambda_{2}, \alpha\right)}{2 \sqrt{2 \pi} \sqrt{1+\lambda_{2}^{2}}} .
\end{aligned}
$$


Now on substituting (3.5), (3.6) and (3.7) in (3.4), we get (3.3).

RESUlt 3.4. The p.d.f. of $\operatorname{ETPSND}\left(\lambda_{1}, \lambda_{2}, \alpha\right)$ is bimodal with unimodes in the regions: $z \in(-\infty, 0)$ and $z \in[0, \infty)$ subject to the conditions given below, in which for $i=0,1$ and $j=1,2$,

$$
a_{i j}=\frac{D^{i}\left(\lambda_{1}, \lambda_{2}, \alpha\right)\left(\lambda_{j} z\right)^{1-i} f\left(\lambda_{j}^{i} z\right)}{\left[1+D\left(\lambda_{1}, \lambda_{2}, \alpha\right) F\left(\lambda_{j} z\right)\right]^{i+1}} .
$$

Region $z \in(-\infty, 0)$ : (i) For all $\alpha \geq 0$ for which either $\lambda_{1} \leq 0$ or $\lambda_{1}>0$ with $\left|a_{01}\right|<a_{11}$,

(ii) For all $\alpha<0$ for which either $\lambda_{1} \leq 0$ with $\left|a_{01}\right|<a_{11}$ or $\lambda_{1}>0$ with $\lambda^{2} f\left(\lambda_{1} z\right)\left(a_{01}+a_{11}\right)>-1$.

Region $z \in[0, \infty)$ : (i) For all $\alpha \geq 0$ for which either $\lambda_{2}>0$ or $\lambda_{2} \leq 0$ with $\left|a_{02}\right|<a_{12}$,

(ii) For all $\alpha<0$ for which either $\lambda_{2}>0$ with $\left|a_{12}\right|<a_{02}$ or $\lambda_{2} \leq 0$ with $\lambda_{2}^{2} f\left(\lambda_{2} z\right)\left(a_{02}+a_{12}\right)>-1$.

Proof. In order to show that there exists unimodes in regions of $z \in$ $(-\infty, 0)$ and $z \in[0, \infty)$, it is enough to show that the second derivative of $h\left(z ; \lambda_{1}, \lambda_{2}, \alpha\right)$ is negative for all $\alpha, \lambda_{1}$ and $\lambda_{2}$ in the respective regions.

For $z \in(-\infty, 0)$, we have

$$
\frac{d^{2}}{d z^{2}}\left\{\log \left[h\left(z ; \lambda_{1}, \lambda_{2}, \alpha\right)\right]\right\}=-1-\lambda_{1}^{2} f\left(\lambda_{1} z\right)\left[a_{00}+a_{10}\right]
$$

and for $z \in[0, \infty)$, we have

$$
\frac{d^{2}}{d z^{2}}\left\{\log \left[h\left(z ; \lambda_{1}, \lambda_{2}, \alpha\right)\right]\right\}=-1-\lambda_{2}^{2} f\left(\lambda_{2} z\right)\left[a_{01}+a_{11}\right] .
$$

Note that for all $\alpha, 1+D\left(\lambda_{1}, \lambda_{2}, \alpha\right) F\left(\lambda_{j} z\right)$ is positive for each $j=1,2$. Now for the region $z \in(-\infty, 0), a_{01}$ is positive or negative according to whether the value of $\lambda_{1}$ is negative or positive and $a_{11}$ is positive or negative according to whether the value of $\alpha$ is positive or negative. If $\alpha \geq 0$, then (3.8) is negative either for $\lambda_{1} \leq 0$ or for $\lambda_{1}>0$ if $a_{01}+a_{11}>0$ and for $\alpha<0,(3.8)$ is negative either for $\lambda_{1}<0$ provided $a_{01}+a_{11}>0$ or for $\lambda_{1}>0$ with $\lambda_{1}^{2} f\left(\lambda_{1} z\right)\left(a_{01}+a_{11}\right)>-1$. Hence the p.d.f. given in (2.1) is log-concave and thus unimodal under these cases.

Now for the region $z \in[0, \infty), a_{02}$ is positive or negative according to whether the value of $\lambda_{2}$ is positive or negative and $a_{12}$ is positive or negative according to whether the value of $\alpha$ is positive or negative. If $\alpha \geq 0$, then (3.9) is negative either for $\lambda_{2}>0$ or for $\lambda_{2}<0$ if $a_{02}+a_{12}>0$. Now for $\alpha<0$, (3.9) is negative either for $\lambda_{2}>0$ if $a_{02}+a_{12}>0$ or $\lambda_{2}<0$ if $\lambda_{2}^{2} f\left(\lambda_{2} z\right)\left(a_{02}+a_{12}\right)>-1$. Thus the p.d.f. given in (2.1) is log-concave and hence unimodal under these conditions.

As a consequence of Result 3.4 we obtain the following result. 
RESULT 3.5. The p.d.f. of $\operatorname{ETPSND}\left(\lambda_{1}, \lambda_{2}, \alpha\right)$ is plurimodal in the regions: $z \in(-\infty, 0)$ and $z \in[0, \infty)$ is as given below:

Region $z \in(-\infty, 0)$ : (i) For all $\alpha \geq 0$ and $\lambda_{1}>0$ such that $\left|a_{01}\right|>a_{11}$.

(ii) For all $\alpha<0, \lambda_{1} \leq 0$ such that $\left|a_{11}\right|>a_{01}$ or $\lambda_{1}>0$ and $\lambda^{2} f\left(\lambda_{1} z\right)\left(a_{01}+\right.$ $\left.a_{11}\right)<-1$.

Region $z \in[0, \infty)$ : (i) For all $\alpha \geq 0, \lambda_{2} \leq 0$ such that $\left|a_{02}\right|>a_{12}$,

(ii) For all $\alpha<0$ for which either $\lambda_{2}>0$ with $\left|a_{12}\right|>a_{02}$ or $\lambda_{2} \leq 0$ with $\lambda_{2}^{2} f\left(\lambda_{2} z\right)\left(a_{02}+a_{12}\right)<-1$.

\section{Location-scale extension}

In this section we consider the location-scale extension of the $\operatorname{ETPSND}\left(\lambda_{1}, \lambda_{2}, \alpha\right)$ and discuss some of its important properties. Let $Z$ follow $\operatorname{ETPSND}\left(\lambda_{1}, \lambda_{2}, \alpha\right)$, then $X=\mu+\sigma Z$ is said to have a location scale extension of the two-piece skew normal distribution with location parameter $\mu$, scale parameter $\sigma$ and shape parameters $\lambda_{1}, \lambda_{2}$ and $\alpha$, which is denoted as $\operatorname{ETPSND}^{L S}\left(\mu, \sigma ; \lambda_{1}, \lambda_{2}, \alpha\right)$. The p.d.f. of $\mathrm{X}$ is given by

$$
\begin{aligned}
h\left(x ; \mu, \sigma, \lambda_{1}, \lambda_{2}, \alpha\right) & \\
= & \left\{\begin{aligned}
& \frac{2}{(\alpha+2) \sigma} f\left(\frac{x-\mu}{\sigma}\right) \\
& \quad \times\left[1+D\left(\lambda_{1}, \lambda_{2}, \alpha\right) F\left(\lambda_{1} \frac{x-\mu}{\sigma}\right)\right], x<\mu \\
& \frac{2}{(\alpha+2) \sigma} f\left(\frac{x-\mu}{\sigma}\right) \\
& \times\left[1+D\left(\lambda_{1}, \lambda_{2}, \alpha\right) F\left(\lambda_{2} \frac{x-\mu}{\sigma}\right)\right], x \geq \mu,
\end{aligned}\right.
\end{aligned}
$$

in which $\mu, \lambda_{1}, \lambda_{2} \in R, \sigma>0$ and $\alpha \geq-1$.

Clearly we have the following special cases.

(i) $\operatorname{ETPSND}^{L S}\left(\mu, \sigma ; \lambda_{1}, \lambda_{2}, 0\right), \operatorname{ETPSND}^{L S}(\mu, \sigma ; 0,0, \alpha)$ or the limiting case of the $\operatorname{ETPSND}^{L S}\left(\mu, \sigma ; \lambda_{1}, \lambda_{2}, \alpha\right)$ when $\lambda_{1} \rightarrow-\infty, \lambda_{2} \rightarrow \infty$, is the normal distribution with parameters $\mu$ and $\sigma^{2}$,

(ii) the limiting case of the $\operatorname{ETPSND}^{L S}\left(\mu, \sigma ; \lambda_{1}, \lambda_{2}, \alpha\right)$ when $\alpha \rightarrow \infty$ is the

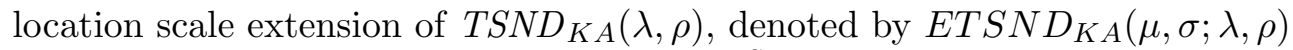

(iii) the limiting case of the $\operatorname{ETPSND}^{L S}(\mu, \sigma ; \lambda, \lambda, \alpha)$ when $\alpha \rightarrow \infty$ is the location scale extension of $\operatorname{TSND}_{K}(\lambda)$, denoted by $\operatorname{ETSN} D_{K}(\mu, \sigma ; \lambda)$

(iv) the $\operatorname{ETPSND}^{L S}(\mu, \sigma ; \lambda, \lambda, \alpha)$ is the location scale extension of $\operatorname{GMNSND}(\lambda, \alpha)$, denoted by $\operatorname{EGMNSND}(\mu, \sigma ; \lambda, \alpha)$ which reduces to the location scale extension of $\operatorname{SND}(\lambda)$, denoted by $\operatorname{ESND}(\mu, \sigma ; \lambda)$ when $\alpha=-1$ and

(v) the limiting case of the $\operatorname{ETPSND}^{L S}\left(\mu, \sigma ; \lambda_{1}, \lambda_{2}, \alpha\right)$ as $\lambda_{1} \rightarrow \infty, \lambda_{2} \rightarrow \infty$ and $\alpha \rightarrow \infty$ or $\lambda_{1} \rightarrow-\infty, \lambda_{2} \rightarrow-\infty$ and $\alpha \rightarrow \infty$ is the half normal distribution.

We obtain the following properties of $\operatorname{ETPSND}^{L S}\left(\mu, \sigma ; \lambda_{1}, \lambda_{2}, \alpha\right)$ with p.d.f. (4.1) in a similar approach as we did in earlier sections. 
RESUlt 4.1. The characteristic function $\phi_{X}(t)$ of a random variable $X$ following $\operatorname{ETPSND}^{L S}\left(\mu, \sigma ; \lambda_{1}, \lambda_{2}, \alpha\right)$ is the following, in which $k_{j}=\lambda_{j} i t, \delta_{j}=$ $\frac{\lambda_{j}}{\sqrt{1+\lambda_{j}^{2}}}$ for $j=1,2$. For $i^{2}=-1$ and $t \in R$,

$$
\begin{aligned}
\phi_{X}(t)= & \frac{2}{(\alpha+2)} e^{i \mu t-t^{2} \sigma^{2} / 2}\left[1+D\left(\lambda_{1}, \lambda_{2}, \alpha\right) F\left(i \delta_{1} \sigma t\right)\right] \\
& -\frac{2}{(\alpha+2)} D\left(\lambda_{1}, \lambda_{2}, \alpha\right) e^{i \mu t-t^{2} \sigma^{2} / 2}\left[\xi_{k_{1} \sigma}\left(-i t \sigma, \lambda_{1}\right)-\xi_{k_{2} \sigma}\left(-i t \sigma, \lambda_{2}\right)\right] .
\end{aligned}
$$

RESUlt 4.2. The distribution function $H_{1}^{*}(t)$ of a random variable $X$ following $\operatorname{ETPSND}^{L S}\left(\mu, \sigma ; \lambda_{1}, \lambda_{2}, \alpha\right)$ is the following,

$$
H_{1}^{*}(t)=\frac{2}{(\alpha+2)}\left\{\begin{array}{c}
F\left(\frac{t-\mu}{\sigma}\right)+\frac{D\left(\lambda_{1}, \lambda_{2}, \alpha\right)}{2} \\
\quad \times\left[F\left(\frac{t-\mu}{\sigma}\right)-2 \xi\left(\left(\frac{t-\mu}{\sigma}\right), \lambda_{1}\right)\right], \quad t<\mu \\
F\left(\frac{t-\mu}{\sigma}\right)+\frac{D\left(\lambda_{1}, \lambda_{2}, \alpha\right)}{2} \\
\times\left[F\left(\frac{t-\mu}{\sigma}\right)-2 \xi\left(\left(\frac{t-\mu}{\sigma}\right), \lambda_{2}\right)\right. \\
\left.-\frac{\tan ^{-1}\left(\lambda_{1}\right)}{\pi}+\frac{\tan ^{-1}\left(\lambda_{2}\right)}{\pi}\right],
\end{array} \quad t \geq \mu .\right.
$$

RESUlt 4.3. The reliability function $R_{1}^{*}(t)$ of a random variable $X$ following $\operatorname{ETPSND}^{L S}\left(\mu, \sigma ; \lambda_{1}, \lambda_{2}, \alpha\right)$ is the following,

$$
R_{1}^{*}(t)=1-\frac{2}{(\alpha+2)}\left\{\begin{aligned}
F\left(\frac{t-\mu}{\sigma}\right)+\frac{D\left(\lambda_{1}, \lambda_{2}, \alpha\right)}{2} \\
\quad \times\left[F\left(\frac{t-\mu}{\sigma}\right)-2 \xi\left(\frac{t-\mu}{\sigma}, \lambda_{1}\right)\right], \quad t<\mu \\
F\left(\frac{t-\mu}{\sigma}\right)+\frac{D\left(\lambda_{1}, \lambda_{2}, \alpha\right)}{2} \\
\quad \times\left[F\left(\frac{t-\mu}{\sigma}\right)-2 \xi\left(\frac{t-\mu}{\sigma}, \lambda_{2}\right)\right] \\
-\frac{D\left(\lambda_{1}, \lambda_{2}, \alpha\right)}{2} \\
\times\left[\frac{-1}{\pi} \tan ^{-1}\left(\lambda_{1}\right)+\frac{1}{\pi} \tan ^{-1}\left(\lambda_{2}\right)\right], \quad t \geq \mu .
\end{aligned}\right.
$$


RESULT 4.4. The failure rate $r_{1}(t)$ of a random variable $X$ following $\operatorname{ETPSND}^{L S}\left(\mu, \sigma ; \lambda_{1}, \lambda_{2}, \alpha\right)$ is the following,

$$
\begin{aligned}
& r_{1}(t) \\
& =\left\{\begin{array}{cc}
\frac{2 f\left(\frac{t-\mu}{\sigma}\right)\left[1+D\left(\lambda_{1}, \lambda_{2}, \alpha\right) F\left(\lambda_{1} \frac{t-\mu}{\sigma}\right)\right]}{\sigma(\alpha+2)-2 \sigma\left(F\left(\frac{t-\mu}{\sigma}\right)+D\left(\lambda_{1}, \lambda_{2}, \alpha\right)\left[F\left(\frac{t-\mu}{\sigma}\right)-2 \xi\left(\frac{t-\mu}{\sigma}, \lambda_{1}\right)\right]\right)}, & t<\mu \\
\frac{2 f\left(\frac{t-\mu}{\sigma}\right)\left[1+D\left(\lambda_{1}, \lambda_{2}, \alpha\right) F\left(\lambda_{2} \frac{t-\mu}{\sigma}\right)\right]}{\sigma(\alpha+2)-2 \sigma\left(F\left(\frac{t-\mu}{\sigma}\right)+D\left(\lambda_{1}, \lambda_{2}, \alpha\right)\left[F\left(\frac{t-\mu}{\sigma}\right)-2 \xi\left(\frac{t-\mu}{\sigma}, \lambda_{2}\right)-\frac{1}{\pi} \tan ^{-1}\left(\lambda_{1}\right)+\frac{1}{\pi} \tan ^{-1}\left(\lambda_{2}\right)\right]\right)} & t \geq \mu .
\end{array}\right.
\end{aligned}
$$

\section{Estimation}

Let $X_{1}, X_{2}, \ldots, X_{n}$ be a random sample of size $n$ from $\operatorname{ETPSND}^{L S}\left(\mu, \sigma ; \lambda_{1}, \lambda_{2}, \alpha\right)$ with p.d.f. (4.1). Let $X_{(1)}, X_{(2)}, \ldots, X_{(n)}$ be the ordered sample. Assume $X_{(r)}<\mu<X_{(r+1)}$, for a particular $r=1,2, \ldots, n$. Then the log-likelihood function $l(\underline{\theta})=l\left(\mu, \sigma ; \lambda_{1}, \lambda_{2}, \alpha\right)$ of the sample is the following, in which $\Sigma_{I_{j}}$, denotes the summation over the set $I_{j}$ such that $I_{1}=\left\{i: X_{(i)}<\mu\right.$, for $i=1,2, \ldots, r\}$ and $I_{2}=\left\{i: X_{(i)} \geq \mu\right.$, for $\left.i=r+1, \ldots, n\right\}$.

$$
\begin{aligned}
l(\underline{\theta})= & n \ln \left(\frac{2}{\sigma(\alpha+2)}\right) \\
& +\sum_{I_{1}} \ln f\left(\frac{x_{i}-\mu}{\sigma}\right)\left[1+D\left(\lambda_{1}, \lambda_{2}, \alpha\right) F\left(\frac{\lambda_{1}\left(x_{i}-\mu\right)}{\sigma}\right)\right] \\
& +\sum_{I_{2}} \ln f\left(\frac{x_{i}-\mu}{\sigma}\right)\left[1+D\left(\lambda_{1}, \lambda_{2}, \alpha\right) F\left(\frac{\lambda_{2}\left(x_{i}-\mu\right)}{\sigma}\right)\right] .
\end{aligned}
$$

On differentiating (5.1) with respect to the parameters $\mu, \sigma, \lambda_{1}, \lambda_{2}$ and $\alpha$ and equating to zero, we obtain the following likelihood equations, in which $u_{i}=\frac{x_{i}-\mu}{\sigma}$ for $i=1,2, \ldots, n, D_{j}^{\prime}\left(\lambda_{1}, \lambda_{2}, \alpha\right)=\frac{d}{d \lambda_{k}} D\left(\lambda_{1}, \lambda_{2}, \alpha\right)$ and for $j=1,2$ and $k=0,1 \beta_{j}\left(x_{i} ; \lambda_{1}, \lambda_{2}, \alpha\right)=1+D\left(\lambda_{1}, \lambda_{2}, \alpha\right) F\left(\lambda_{i} \frac{x_{i}-\mu}{\sigma}\right), \gamma_{j k}\left(x_{i}\right)=\frac{f^{k}\left(\lambda_{j}\right) F^{1-k}\left(\lambda_{j} u_{i}\right)}{\beta_{j}\left(x_{i} ; \lambda_{1}, \lambda_{2}, \alpha\right)}$.

$$
\begin{aligned}
\sum_{I_{1}} & \frac{\left(x_{i}-\mu\right)}{\sigma}+\sum_{I_{2}} \frac{\left(x_{i}-\mu\right)}{\sigma} \\
= & D\left(\lambda_{1}, \lambda_{2}, \alpha\right)\left[\lambda_{1} \sum_{I_{1}} \gamma_{11}\left(x_{i}\right)+\lambda_{2} \sum_{I_{2}} \gamma_{21}\left(x_{i}\right)\right] \\
\frac{n}{2 \sigma^{2}}= & \frac{1}{2} \sum_{I_{1}} \frac{\left(x_{i}-\mu\right)^{2}}{\sigma^{4}}+\frac{1}{2} \sum_{I_{2}} \frac{\left(x_{i}-\mu\right)^{2}}{\sigma^{4}} \\
& \quad-\frac{D\left(\lambda_{1}, \lambda_{2}, \alpha\right)}{2 \sigma^{3}}\left(\lambda_{1} \sum_{I_{1}} \gamma_{11}\left(x_{i}\right)\left(x_{i}-\mu\right)+\lambda_{2} \sum_{I_{2}} \gamma_{21}\left(x_{i}\right)\left(x_{i}-\mu\right)\right)
\end{aligned}
$$


(5.4)

$$
\begin{gathered}
\frac{D\left(\lambda_{1}, \lambda_{2}, \alpha\right)}{\sigma} \sum_{I_{1}} \gamma_{11}\left(x_{i}\right)\left(x_{i}-\mu\right)+\sum_{I_{1}} \gamma_{10}\left(x_{i}\right) D_{1}^{\prime}\left(\lambda_{1}, \lambda_{2}, \alpha\right) \\
+\sum_{I_{1}} D_{1}^{\prime}\left(\lambda_{1}, \lambda_{2}, \alpha\right) \beta_{1}\left(x_{i} ; \lambda_{1}, \lambda_{2}, \alpha\right)=0 \\
\frac{D\left(\lambda_{1}, \lambda_{2}, \alpha\right)}{\sigma} \sum_{I_{1}} \gamma_{21}\left(x_{i}\right)\left(x_{i}-\mu\right)+\sum_{I_{1}} \gamma_{20}\left(x_{i}\right) D_{2}^{\prime}\left(\lambda_{1}, \lambda_{2}, \alpha\right) \\
+\sum_{I_{1}} D_{2}^{\prime}\left(\lambda_{1}, \lambda_{2}, \alpha\right) \beta_{2}\left(x_{i} ; \lambda_{1}, \lambda_{2}, \alpha\right)=0
\end{gathered}
$$

and

$$
D\left(\lambda_{1}, \lambda_{2}, \alpha\right) \sum_{I_{1}} \gamma_{10}+D\left(\lambda_{1}, \lambda_{2}, \alpha\right) \sum_{I_{2}} \gamma_{20}=\frac{n \alpha}{(\alpha+2)}
$$

Solving the non-linear system of equations (5.2) to (5.6) by simultaneous solution method using mathematical software such as MATHCAD, MATLAB, MATHEMATICA etc., the maximum likelihood estimates (MLE) of the parameters of $\operatorname{ETPSND}^{L S}\left(\mu, \sigma ; \lambda_{1}, \lambda_{2}, \alpha\right)$ can be obtained.

\section{Applications}

For illustrating the usefulness of the $\operatorname{ETPSND}^{L S}\left(\mu, \sigma ; \lambda_{1}, \lambda_{2}, \alpha\right)$, in this section we have considered the IQ data set for 52 non-white males and 87 white males hired by a large insurance company in 1971 given in Roberts (1988).

Data set 1: 9110210011712211597109108104108118103123123 103106102118100103107108107979511910210810310211299116114 10211110412210311110191991219710910610210410795.

Data set 2: 8594949798100100101102102103103103103104104 106106106106106107107108108108108108108108109109111111112 112112112112112112112112112113113113113113113113113114114 115116116116116117117117118118118119120120120121121121122 122122122122122124124125129131132135136140.

Here we have fitted the proposed model to these two data sets, non-white males and white males, and compared results with the existing models such as the normal distribution $N(\mu, \sigma), \quad \operatorname{ESND}(\mu, \sigma, \lambda), \quad \operatorname{ETSND}_{K}(\mu, \sigma ; \lambda, \rho) \quad$ and $\operatorname{ETPSND}^{L S}\left(\mu, \sigma ; \lambda_{1}, \lambda_{2}, \alpha\right)$. For model comparison, we have computed some wellknown information criterion-such as Akaike's Information Criterion (AIC), the Bayesian Information Criterion (BIC) and the corrected Akaike's Information Criterion (AICc). For numerical evaluation, we have used MATHCAD software and the results obtained are included in Table 1 for the case of Data set 1 and in Table 2 for the case of Data set 2 along with the log-likelihood $(l)$.

In light of the computed values listed in Tables 1 and 2 , it can be seen that the $\operatorname{ETPSND}^{L S}\left(\mu, \sigma ; \lambda_{1}, \lambda_{2}, \alpha\right)$ gives the best results compared to other existing models. 
Table 1. MLE of the parameters of the $\operatorname{ETPSND}^{L S}\left(\mu, \sigma ; \lambda_{1}, \lambda_{2}, \alpha\right)$ and computed values of the $l$, AIC, BIC and AICc values in the case of various fitted models to data set 1 .

\begin{tabular}{|c|c|c|c|c|}
\hline Distribution: & $\begin{array}{c}N \\
(\mu, \sigma)\end{array}$ & $\begin{array}{c}E S N D \\
(\mu, \sigma, \lambda)\end{array}$ & $\begin{array}{c}E^{2}{ }^{2} D_{K} \\
(\mu, \sigma ; \lambda, \rho)\end{array}$ & $\begin{array}{c}E T P S N D^{L S} \\
\left(\mu, \sigma ; \lambda_{1}, \lambda_{2}, \alpha\right)\end{array}$ \\
\hline$\hat{\mu}$ & 106.653 & 98.79 & 104.64 & 102.45 \\
$\hat{\sigma}$ & 8.229 & 11.38 & 8.9 & 9.8 \\
$\hat{\lambda}$ & - & 1.71 & 0.8 & $2.54,-0.457$ \\
$\hat{\alpha}$ & - & - & - & 1.66 \\
$\hat{\rho}$ & - & - & -0.04 & - \\
$l$ & -183.374 & -182.423 & -182 & -177 \\
$\mathrm{AIC}$ & 371 & 371 & 370 & 365 \\
$\mathrm{BIC}$ & 375 & 377 & 376 & 374 \\
$\mathrm{AICc}$ & 370.99 & 371.34 & 370.73 & 366 \\
\hline
\end{tabular}

Table 2. MLE of the parameters of the $\operatorname{ETPSND}^{L S}\left(\mu, \sigma ; \lambda_{1}, \lambda_{2}, \alpha\right)$ and computed values of the $l, \mathrm{AIC}, \mathrm{BIC}$ and AICc values in the case of various fitted models to data set 2 .

\begin{tabular}{|c|c|c|c|c|}
\hline Distribution: & $\begin{array}{c}N \\
(\mu, \sigma)\end{array}$ & $\begin{array}{c}E S N D \\
(\mu, \sigma, \lambda)\end{array}$ & $\begin{array}{c}\text { ETSND }_{K} \\
(\mu, \sigma ; \lambda, \rho)\end{array}$ & $\begin{array}{c}E T P S N D^{L S} \\
\left(\mu, \sigma ; \lambda_{1}, \lambda_{2}, \alpha\right)\end{array}$ \\
\hline$\hat{\mu}$ & 112.86 & 105.78 & 107.1 & 108.3 \\
$\hat{\sigma}$ & 9.58 & 11.94 & 10.9 & 11.5 \\
$\hat{\lambda}$ & - & 1.14 & 0.899 & $0.49,-0.0098$ \\
$\hat{\alpha}$ & - & - & - & -0.91 \\
$\hat{\rho}$ & - & - & 0.9 & - \\
$l$ & -319.6 & -319.29 & -319 & -314 \\
$\mathrm{AIC}$ & 643.2 & 644.57 & 647 & 638 \\
$\mathrm{BIC}$ & 648.14 & 651.97 & 657 & 647 \\
$\mathrm{AICc}$ & 643.35 & 644.86 & 647.32 & 639.01 \\
\hline
\end{tabular}

\section{Acknowledgements}

The authors are grateful to the Editor, Associate Editor and the anonymous referee for carefully reading the paper and for the valuable comments and suggestions. The second author is thankful to the Department of Science and Technology, Ministry of Science and Technology, New Delhi for financial support (IF 110142).

\section{REFERENCES}

Arellano-Valle, R. B., Gomez, H. W. and Quintana, F. A. (2004). A new class of skew normal distribution, Comm. Statist.-Theory Meth., 33(7), 1465-1480.

Arnold, B. C. and Beaver, R. J. (2002). Skewed multivariate models related to hidden truncation and/or selective reporting (with discussion), Test, 11, 7-54.

Azzalini, A. (1985). A class of distributions which includes the normal ones, Scand. J. Statist., 12, $171-178$.

Azzalini, A. (1986). Further results on a class of distributions which includes the normal, Statistica, 46, 199-208. 
Azzalini, A. and Dalla Valle, A. (1996). The multivariate skew normal distribution, Biometrika, 83, $715-726$.

Branco, M. and Dey, D. (2001). A general class of multivariate elliptical distributions, J. Mult. Anal., 79, 99-113.

Henze, N. (1986). A probabilistic representation of the skew normal distribution, Scand. J. Statist., 13, 271-275.

Jamalizadeh, A., Behboodian, J. and Balakrishnan, N. (2008). A two-parameter generalized skew-normal distribution, Stat. Probab. Lett., 78, 1722-1728.

Jamalizadeh, A., Arabpour, A. R. and Balakrishnan, N. (2009). A generalized skew two-piece skew-normal distribution, Statist. Pap., 79, 99-113.

Kim, H. J. (2005). On a class of two-piece skew-normal distributions, Statistics, 39(6), 537-553.

Kumar, C. S. and Anusree, M. R. (2011). On a generalized mixture of standard normal and skew normal distributions, Stat. Probab. Lett., 81, 1813-1821.

Kumar, C. S. and Anusree, M. R. (2013a). A generalized two-piece skew normal distribution and some of its properties, Statistics, 47(6), 1370-1380.

Kumar, C. S. and Anusree, M. R. (2013b). On an extended version of skew generalized normal distribution and some of its properties, Comm. Statist.-Theory Meth., ISSN: 0361-0926, doi:10.1080/03610926.2012.739251.

Kumar, C. S. and Anusree, M. R. (2014a). On a modified class of generalized skew normal distribution, Sou. Afri. Statist. J., 48(1), 111-124.

Kumar, C. S. and Anusree, M. R. (2014b). On a modified generalized skew normal distribution and some of its properties, J. Statist.-Theory Pract., ISSN: 1559-8616, doi:10.1080/ 15598608.2014.935617.

Mudholkar, G. S. and Hutson, A. D. (2000). The epsilon-skew-normal distribution for analysing near-normal data, J. Statist. Plann. Inf., 83, 291-309.

Roberts, H. V. (1988). Data Analysis for Managers with Minitab, Scientific Press, Redwood City, CA.

Sharafi, M. and Behboodian, J. (2008). The Balakrishnan skew-normal density, Statist. Pap., 49, 769-778. 